\title{
HIGH-SENSITIVITY AND SELECTIVITY DETECTION OF PERMANGANATE IONS BASED ON PIG LIVER-BASED CARBON QUANTUM DOTS
}

\author{
TU, Y. J. ${ }^{1,2,3}-$ TIAN, Y. H. ${ }^{4}-$ YANG, Y. L. ${ }^{1 *}$ \\ ${ }^{I}$ Faculty of Life Science and Technology, Kunming University of Science and Technology, \\ Yunnan Province 650500, China \\ ${ }^{2}$ Faculty of Environmental Science and Engineering, Kunming University of Science and \\ Technology, Yunnan Province 650500, China \\ ${ }^{3}$ Department of Chemical Science and Technology, Kunming University, Yunnan Province \\ 650214, China \\ ${ }^{4}$ School of Metallurgy and Energy Engineering, Kunming University of Science and \\ Technology, Kunming 650500 Yunnan Province, China \\ *Corresponding author \\ e-mail: yilyil8@163.com
}

(Received 26 ${ }^{\text {th }}$ Dec 2018; accepted $3^{\text {rd }}$ May 2019)

\begin{abstract}
In this paper, novel fluorescent carbon quantum dots (CQDs) were successfully prepared from pig liver by a simple one-step hydrothermal method. The prepared CQDs were characterized using TEM, XRD, XPS, FT-IR, UV-Vis, and fluorescence spectroscopy. It was found that the CQDs were uniformly distributed in size and exhibited good excitation dependence and excellent photostability. Fluorescent probe was used for detection of permanganate. This paper also studied the fluorescent staining performance of pig liver-based CQDs on four live/dead bacteria to evaluate the low toxicity and application prospects of the CQDs. Under the optimum experimental conditions, the probe showed a good linear range $\left(\mathrm{R}^{2}=0.9967\right)$ and a low detection limit $(0.06 \mu \mathrm{M})$ in the concentration range of $0.1-50 \mu \mathrm{M}$. The pig liver based-CQDs were proved to be a low toxicity fluorescent dye. These attractive properties indicated that the new CQDs could adapt to various complex $\mathrm{pH}$ environments, and thus had extensive prospect and promising application for detection of permanganate in complex environmental samples.

Keywords: carbon quantum dots, pig liver, bacterial staining, in vivo bio-imaging, $\mathrm{MnO}_{4}$
\end{abstract}

\section{Introduction}

Manganese is an essential trace element of human body. Too much or too little manganese in the body can cause adverse consequences (Teo and Chen, 2001). Manganese deficiency affects the body's reproductive function, lipid metabolism (da Silva et al., 2006), glucose metabolism (Doroschuk et al., 2004) and immune function (Rask et al., 2015), thus causing mental retardation, dysmotility and balance disorders (Gunter et al., 2010), and skeletal dysfunction (Liang et al., 2006). It can also deform the next generation. The excessive amount of manganese in the body can lead to mental symptoms (Zeng et al., 2017). Therefore, the analysis of manganese in food and environment samples is of important practical significance, and it is highly necessary to monitor the level of manganese in body fluids or environmental samples.

Permanganate is usually oxidable, hazardous explosion chemicals, precursor chemicals, and easy to decompose by heating because they all have the same anionspermanganate ions. Permanganate ion has strong oxidation in acidic solution (acidic 
$\mathrm{KMnO}_{4}$ (potassium permanganate)). It also has certain oxidation in alkaline solution, which is purple red in solution. The oxidation of potassium permanganate can be found in both acidity and alkalinity, but it is more obvious in acidity. In recent years, many methods of manganese detection have been developed, including catalytic kinetic spectrophotometric for the determination of trace manganese, but there are few methods for the determination of permanganate ions. A new fluorescence method for the determination of trace manganese was established (Xu et al., 2004), where the concentration range of $\mathrm{MnO}_{4}{ }^{-}$is $1.0 \sim 2.4 \mathrm{mg} / \mathrm{L}$, the maximum excitation wavelength is $278 \mathrm{~nm}$, the maximum emission wavelength is $616 \mathrm{~nm}$, and the fluorescence intensity and concentration show a good linear relationship by transforming the manganese into $\mathrm{MnO}_{4}{ }^{-}$. The results obtained in this method were satisfactory.

Carbon quantum dots (CQDs) are one of the most popular carbon nanomaterials after fullerene, carbon nanotubes and graphene. They were first discovered in 2004 (Wang and $\mathrm{Hu}, 2014$ ) when electrophoresis was used to purify single-walled carbon nanotubes. They have attracted great attention due to their excellent optical properties, such as water solubility, chemical inertia, low toxicity, easy functionalization (Li et al., 2012) and high fluorescence intensity, photobleaching resistance and adjustable luminescence color. They have been widely used in detection of metal ions (Lu et al., 2012), anions (Zhao et al., 2011), small organic molecules (Li et al., 2013) and biological molecules (Deng et al., 2014). The method of detecting metal ions or other environmental pollutants using CQDs as a fluorescent probe has received extensive attention due to its excellent sensitivity and selectivity (Clarkson and Magos, 2006). Zhang and Chen (2014) used folic acid as carbon source and nitrogen source to trace mercury ions by nitrogen-doped carbon dots, and the detection limit was $0.23 \mathrm{~mol} / \mathrm{L}$.

In this study, fluorescent carbon quantum dots (CQDs) were successfully prepared by simple one-step hydrothermal method using pig liver as a carbon source. Since pig liver is a complex that contains a number of organics and biomolecules including fat, proteins, vitamin $\mathrm{B}$, vitamin $\mathrm{C}$, vitamin $\mathrm{E}$, carbohydrates, cholesterol and minerals, it can be useful for doping of multiple hetero atoms in the CQDs without addition of any additives. In the experiment, we found that $\mathrm{MnO}_{4}{ }^{-}$could quench the fluorescence of CQDs, and the quenching degree was related to the concentration of $\mathrm{MnO}_{4}^{-}$. To our knowledge, there is no report used CQDs fluorescent probe from pig liver for detection of $\mathrm{MnO}_{4}^{-}$yet, which provides a new method for sensitive detection of $\mathrm{MnO}_{4}^{-}$. In addition, this method has been successfully applied to detecting $\mathrm{MnO}_{4}{ }^{-}$in environmental water and soil samples.

\section{Experiment}

\section{Instruments and reagents}

Pig liver was purchased from a local supermarket in Kunming (Yunnan, China); all analytical reagents, including potassium permanganate $\left(\mathrm{KMnO}_{4}\right)$, citric acid, disodium hydrogen phosphate $\left(\mathrm{Na}_{2} \mathrm{HPO}_{4}\right), \mathrm{FeCl}_{3} \cdot 6 \mathrm{H}_{2} \mathrm{O}$ and $\mathrm{FeCl}_{2} \bullet 4 \mathrm{H}_{2} \mathrm{O}$, sodium nitrite, sodium nitrate, $\mathrm{KI}, \mathrm{NaCl}, \mathrm{NaF}$, copper sulfate, aluminum sulfate, calcium chloride, $\mathrm{MnSO}_{4}$, $\mathrm{BaCl}_{2}, \mathrm{MgCl}_{2}$ and sodium sulfide (NaS) were purchased from Shanghai Aladdin Biochemical Technology Co., Ltd. (Shanghai, China); and all solutions were prepared from Milli-Q system ultrapure water (18.2 M $\left.2 . \mathrm{cm}, 25^{\circ} \mathrm{C}\right)$.

G9800A fluorospectrophotometer (Agilent Technologies, USA) was used to determine the fluorescence spectrum and its intensity; UV-2600 spectrophotometer 
(Shimadzu, Japan) was used to determine UV spectra; Tecnai G2 F30S-Twin high resolution field emission transmission electron microscope (FEI, Netherlands) was used to detect the particle size and morphology of fluorescent quantum dots; TENSOR 27 Fourier transform infrared spectrometer (Bruker, Germany) was used to determine the infrared spectrum and the structure of the material; D8-advance X-ray powder diffractometer (Bruker, Germany) was used to determine the crystal structure morphology; Thermo Scientific K-Alpha X-ray photoelectron spectrometer (Thermo Fisher Scientific Inc. U.S.A.) was used to analyze the elemental composition ratio and chemical oxidation state; XH-B vortex instrument (Shanghai Hanuo Instrument Co., Ltd., China) was used for vortex mixing; and 80-2 high speed centrifuge (Shanghai Surgical Instrument Factory, China) was used for centrifugal filtration.

\section{Preparation of pig liver-based CQDs}

The pig liver was dried naturally at room temperature and grinded into powders in a mortar. The CQDs of pig liver were prepared by simple hydrothermal method using pig liver powder as carbon source. Five grams of pig liver powder was dispersed in $150 \mathrm{~mL}$ deionized water. Then, the mixture was transferred into a high-pressure autoclave $(200 \mathrm{~mL})$ lined with polytetrafluoroethylene (PTFE), heated at $180{ }^{\circ} \mathrm{C}$ for $10 \mathrm{~h}$, cooled naturally to room temperature, and centrifuged at $13,000 \mathrm{rpm}$ for $20 \mathrm{~min}$ to obtain bright yellow solution, and filtered to remove insoluble substances. In order to obtain pure pig liver-based CQDs, the solution was filtered by $0.22 \mu \mathrm{m}$ membrane. Finally, the obtained pig liver CQDs were stored at $4{ }^{\circ} \mathrm{C}$ for further use. Compared with other synthetic methods, the hydrothermal synthesis of pig liver CQDs is simpler and more feasible. In addition, pig liver as raw material for synthesis is cheap and easily available, and this method is greener and more environmentally friendly.

\section{Fluorescence quenching test for permanganate}

Typically, $50 \mu \mathrm{L}$ of prepared pig liver-based CQDs solution and $1 \mathrm{~mL}$ of $\mathrm{pH} 7.0$ citric acid-disodium hydrogen phosphate buffer solution mixed and diluted to $3.0 \mathrm{~mL}$ with deionized water were placed in a $10 \mathrm{~mL}$ glass centrifuge tube. Then, $2 \mathrm{~mL}$ permanganate solution or sample with a different concentration was added to the centrifuge tube and the vortex was mixed with $30 \mathrm{~s}$. After $3 \mathrm{~min}$, the fluorescence intensity was measured at excitation wavelength $278 \mathrm{~nm}$ and emission wavelength $616 \mathrm{~nm}$ on the fluorescence spectrophotometer, and the fluorescence intensity was recorded. The slit width of excitation and emission was $5 \mathrm{~nm}$. In order to evaluate the effect of coexisting interfering substances on the fluorescence intensity of pig liverbased CQDs and the selectivity of permanganate and other substances or ions (including $\mathrm{FeCl}_{3} \bullet 6 \mathrm{H}_{2} \mathrm{O}$ and $\mathrm{FeCl}_{2} \bullet 4 \mathrm{H}_{2} \mathrm{O}$, sodium nitrite, sodium nitrate, $\mathrm{KI}, \mathrm{NaCl}, \mathrm{NaF}$, copper sulfate, aluminum sulfate, calcium chloride, $\mathrm{MnSO}_{4}, \mathrm{BaCl}_{2}, \mathrm{MgCl}_{2}$ and sodium sulfide), the fluorescence quenching test was carried out under the same experimental conditions. The schematic diagram of detection of $\mathrm{MnO}_{4}{ }^{-}$with pig liver-based CQDs was shown in Figure 1. 


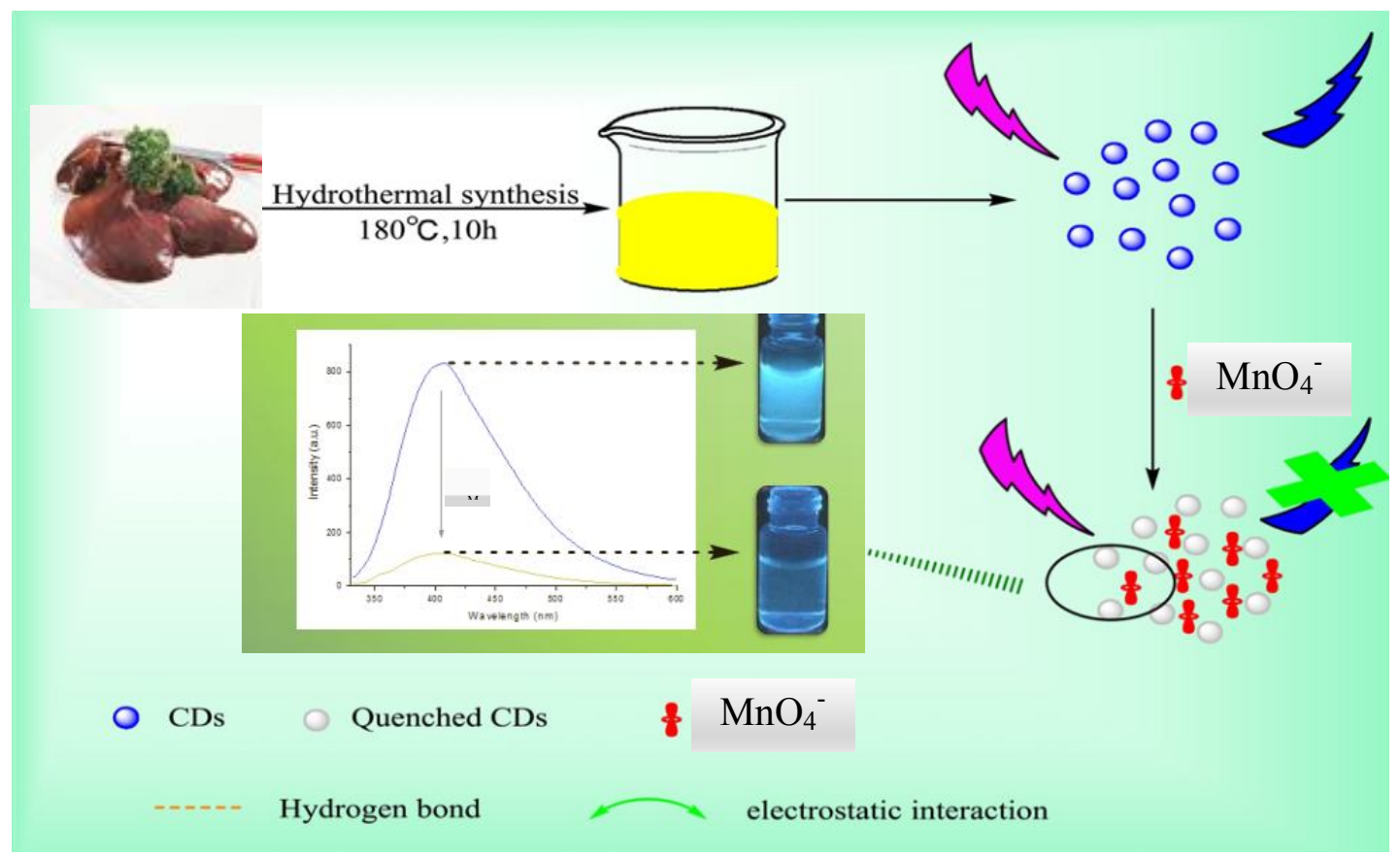

Figure 1. Processing diagram for the synthesis and application of CQDs

\section{Collection and pretreatment of environmental samples}

In this study, water samples were collected directly from our laboratory, Laoyuhe Lake and the domestic wastewater of the community next to a school. All water samples were filtered by simple centrifugal filtration to remove solid particles and suspended solids and then by $0.22 \mu \mathrm{m}$ microporous membrane, and stored at $4{ }^{\circ} \mathrm{C}$. The permanganate ion was added to the samples at a concentration of $0.01 \mathrm{mg} / \mathrm{mL}$ for spiked fluorescence analysis.

Soil samples were collected from the road outside the school after rain and dried under suitable environmental conditions (final moisture content $0.32 \%$ ). One milliliter of methanol solution containing different concentrations of permanganate ions was added to $200 \mathrm{mg}$ soil, mixed for $1 \mathrm{~h}$ on a $50 \mathrm{rpm}$ shaker, and then allowed to stand overnight at room temperature (RT). On the second morning, the mixture was again vortexed and centrifuged for $2 \mathrm{~min}$ at 5,000 rpm and the supernatant was used for fluorescence detection.

\section{Results and discussion \\ Characterization of the CQDs}

The transmission electron microscopy (TEM) technique was used to explore the morphology and particle size distributions of CQDs. As shown in Figure 2, the CQDs present high dispersity, uniform spherical shapes, and a size distribution within the range of 3-10 nm and an average diameter of about $5.0 \mathrm{~nm}$. The surface chemistry of CQDs was studied using Fourier transform infrared (FT-IR) spectroscopy. As shown in Figure 3, the strong absorption peak at $1469 \mathrm{~cm}^{-1}$ reveals the existence of $-\mathrm{COOH}$ stretching vibrations; the peak at $1652 \mathrm{~cm}^{-1}$ reveals the existence of $\mathrm{O}=\mathrm{C}-\mathrm{NH}$ stretching vibrations; and the absorption peak at $3190 \mathrm{~cm}^{-1}$ displays $\mathrm{O}-\mathrm{H}$ stretching vibrations. As shown in Figure 4, the full scan XPS spectra present distinct peaks at 
287.3 and $532.7 \mathrm{eV}$, which are attributed to $\mathrm{C} 1 \mathrm{~s}$ and $\mathrm{O} 1 \mathrm{~s}$, respectively. The result of XPS diagram was consistent with that of FT-IR analysis. Therefore, there should be hydrophilic groups on the surface of pig liver-based CQDs, such as $-\mathrm{COOH},-\mathrm{OH}$, etc., which have good water solubility and broad application prospects.

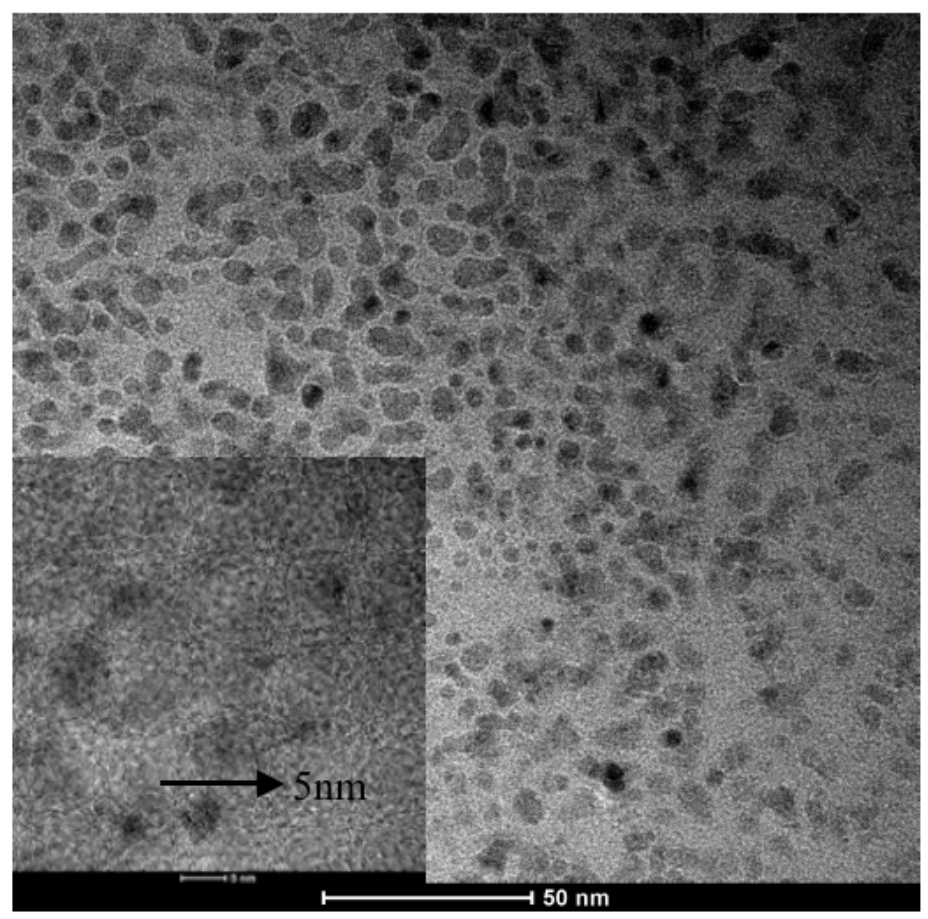

Figure 2. TEM image (inset: HR-TEM image) of the synthesized CQDs

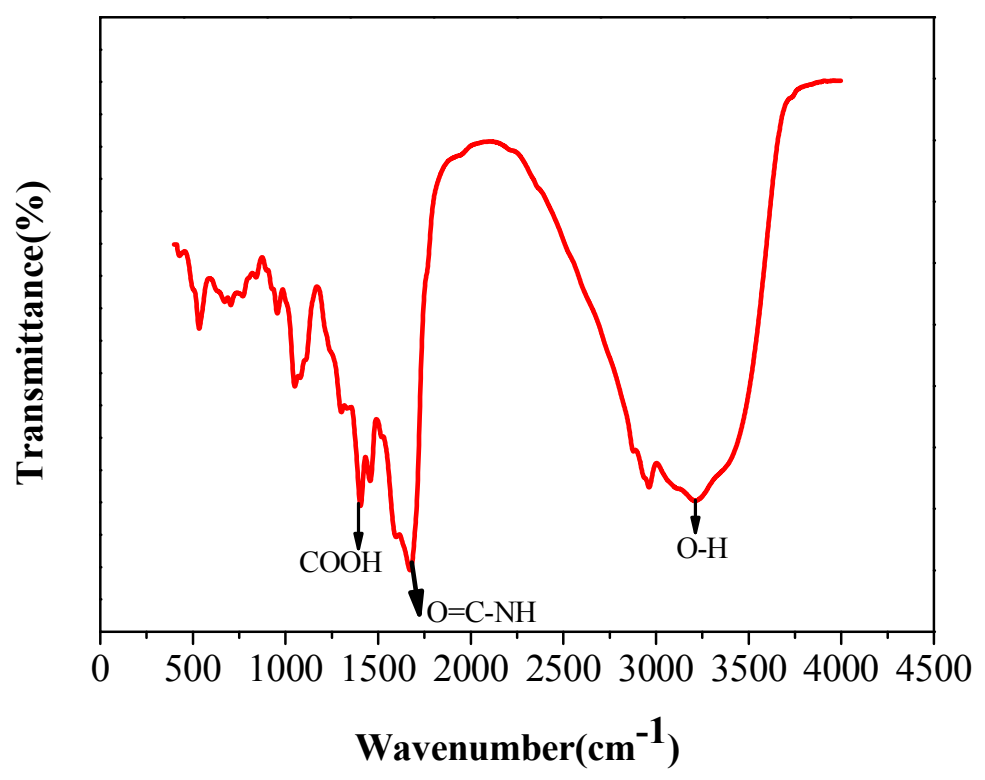

Figure 3. FT-IR spectrum of pig liver-based CQDs 


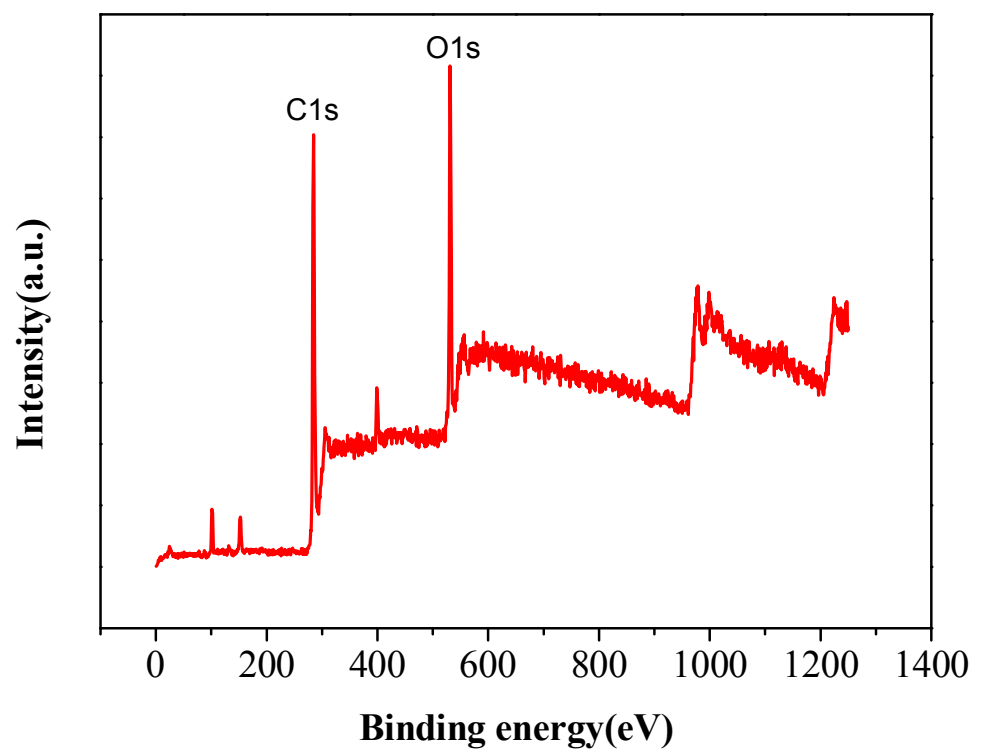

Figure 4. XPS spectra of pig liver-based CQDs

In order to study the optical properties of pig liver-based CQDS, the pig liver-based CQDs were characterized by UV-vis absorption spectrum and fluorescence emission spectrum. The UV-vis absorption spectrum (UV-vis) of pig liver-based CQDs is shown in Figure 5. The prepared pig liver-based CQDs had a weak absorption peak around $258 \mathrm{~nm}$ and an excitation wavelength of $278 \mathrm{~nm}$ at the maximum emission wavelength of $616 \mathrm{~nm}$, as shown in Figure 6, a fluorescence spectrum (FS) and an excitationdependent emission spectrum of pig liver-based CQDs. The results showed that when the excitation wavelength was changed from 300 to $360 \mathrm{~nm}$ in increments of $10 \mathrm{~nm}$, the emission peak shifted toward the long wavelength direction.

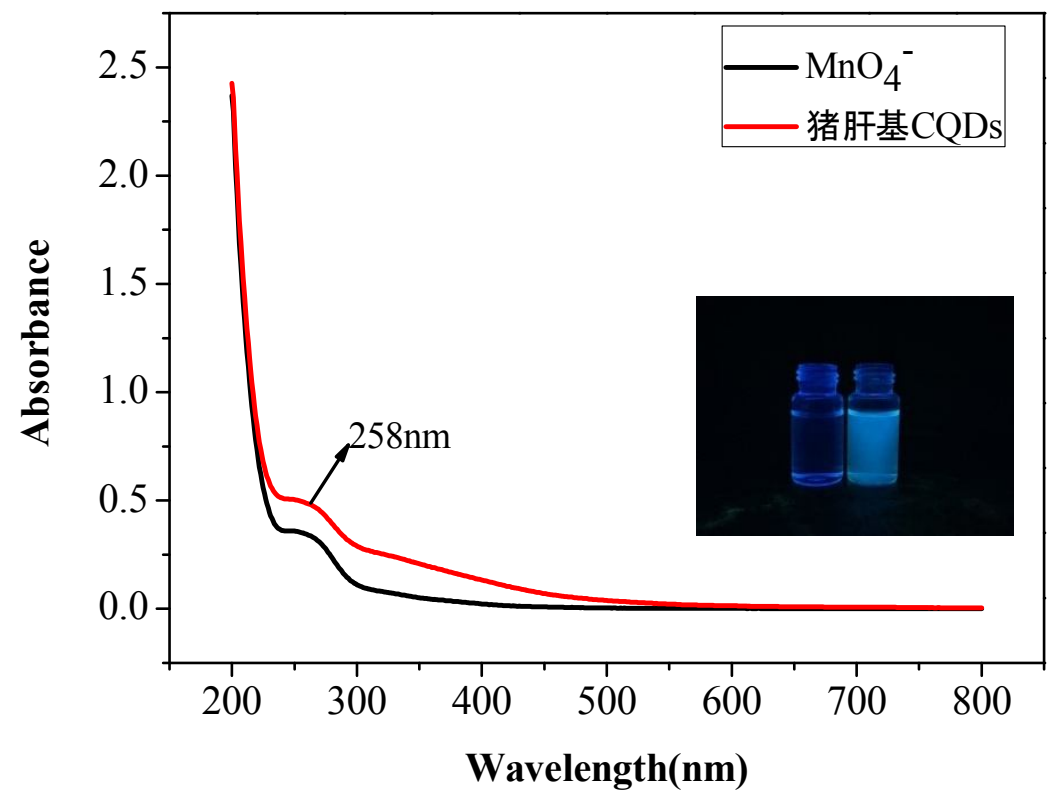

Figure 5. UV-Vis absorption spectra of the synthesized pig liver-based CQDs. (Inset: photographs of pig liver-based CQDs under daylight (left) and UV (365 nm) irradiation (right)) 


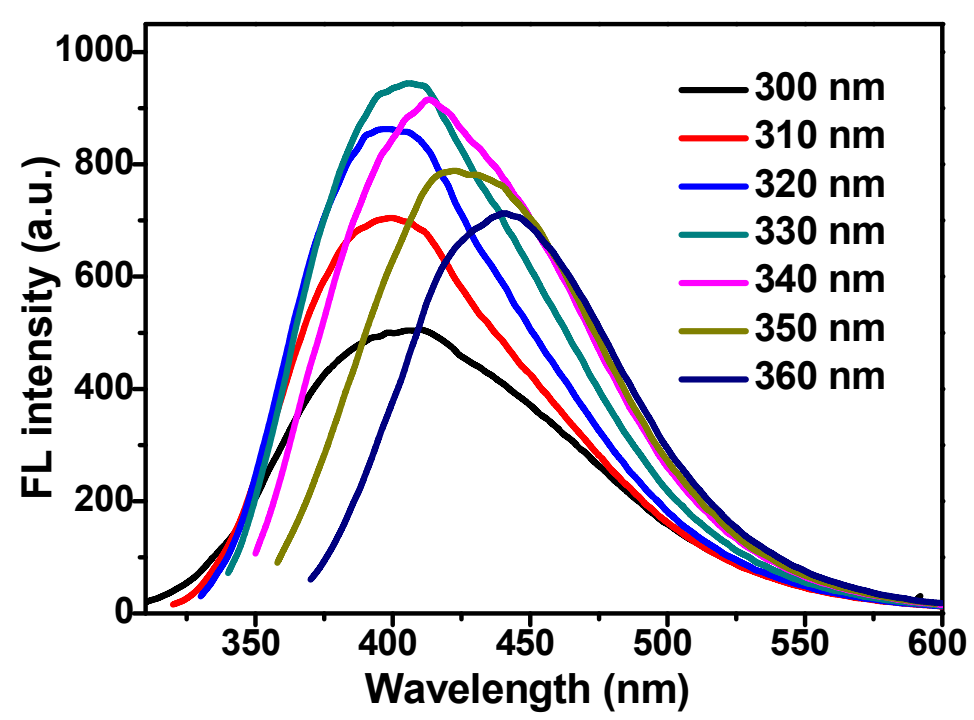

Figure 6. Fluorescence emission spectra of pig liver-based CQDs at different excitation wavelengths

\section{Light stability test of pig liver-based CQDs}

In order to study the stability of pig liver-based CQDs in detecting permanganate, the fluorescence intensity of pig liver-based CQDs at different storage time was studied. As shown in Figure 7A, the fluorescence intensity of the pig liver based-CQDs is substantially constant. In addition, the reaction time increased while the fluorescence intensity of the experimental system remained constant, indicating that the storage time had no effect on the fluorescence intensity of pig liver-based CQDs. The effects of ionic strength and $\mathrm{pH}$ on the fluorescence intensity of pig liver-based CQDs were also studied, as shown in Figure $7 B$ and $C$. It is observed from the figures that with the gradual increase of the concentration of $\mathrm{NaCl}$ solution, the fluorescence intensity only slightly fluctuated without significant change; the $\mathrm{pH}$ of the citric acid-dibasic sodium phosphate buffer changed from 2.0 to 8.0; and the fluorescence intensity of pig liverbased CQDs remain unchanged, this demonstrates that pig liver-based CQDs can be used as fluorescent nanoprobe in any environmental conditions.

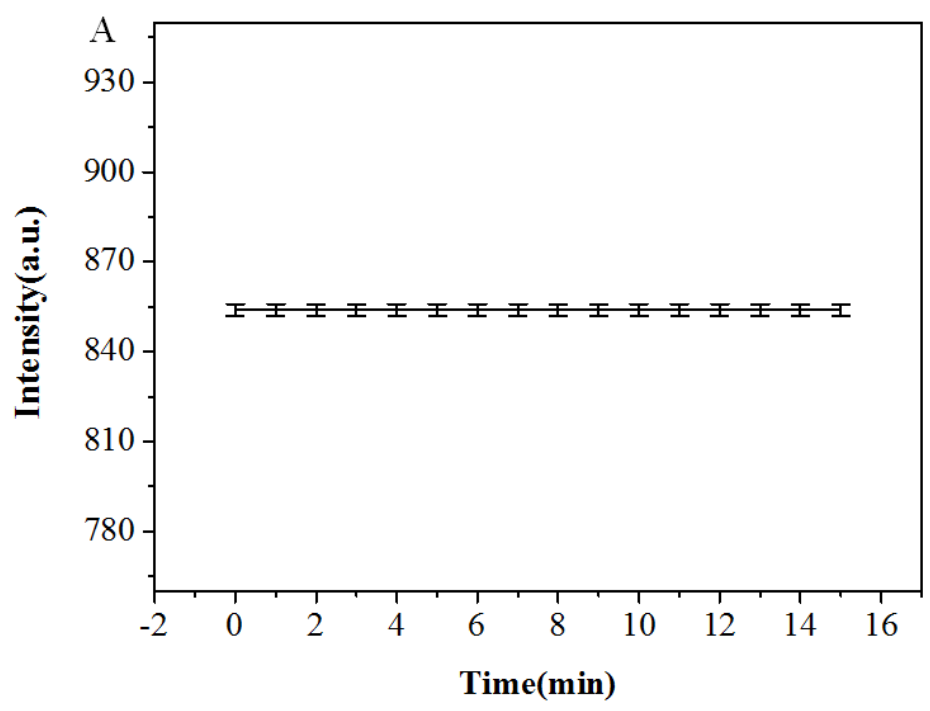



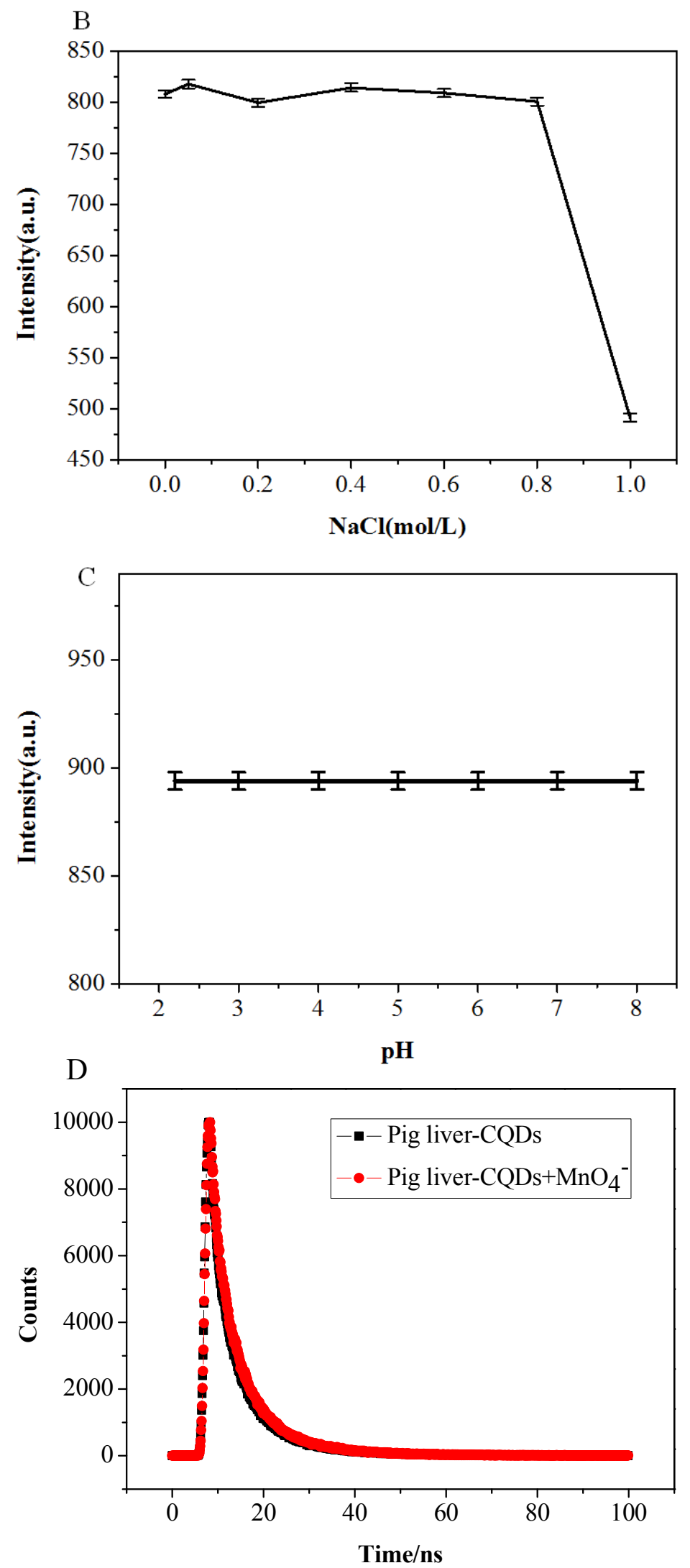

Figure 7. Effects of various conditions on the fluorescence intensity of pig liver-based CQDs: (A) storage time; (B) $\mathrm{NaCl}$ concentration; (C) $\mathrm{pH}$; (D) the Fluorescence lifetime decay curve 


\section{Selectivity}

The selectivity of pig liver based-CQDs was investigated by monitoring the fluorescence intensity of different possible interfering substances such as $\mathrm{MnO}_{4}^{-}, \mathrm{Fe}^{3+}$, $\mathrm{Fe}^{2+}, \mathrm{NO}_{2}, \mathrm{NO}_{3}, \mathrm{I}^{-}, \mathrm{Cl}^{-}, \mathrm{F}^{-}, \mathrm{Na}^{+}, \mathrm{K}^{+}, \mathrm{Cu}^{2+}, \mathrm{Al}^{3+}, \mathrm{Ca}^{2+}, \mathrm{Mn}^{2+}, \mathrm{Ba}^{2+}, \mathrm{Mg}^{2+}$ and $\mathrm{S}^{2-}$ in the presence of different concentrations or ions. As shown in Figure 8, only permanganate ion can effectively quench the fluorescence intensity of CQDs in pig liver, while the quenching effect of other possible interfering substances is negligible. The results show that there may be some quenching relationship between pig liver-based CQDs and permanganate ions, which is worthy of further study. Therefore, there is a high selectivity between permanganate ions and pig liver-based CQDs. This demonstrates that pig liver-based CQDs can be used to detect permanganate ions.

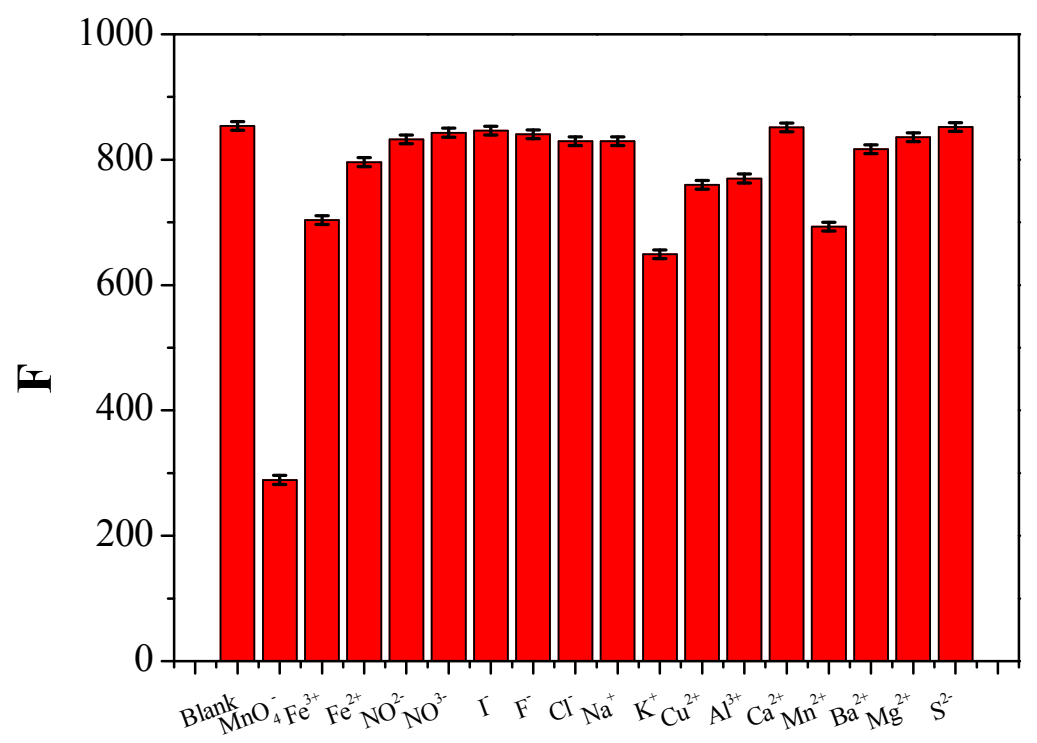

Figure 8. Evaluation of method selectivity against possible interferents

\section{Optimization of experimental conditions}

PH affects not only the fluorescence intensity of pig liver-based CQDs, but also the fluorescence quenching effect of permanganate ions on pig liver-based CQDs. Therefore, the effect of $\mathrm{pH}$ from 2.2 to 8.0 on the experimental system was investigated. As shown in Figure 9A, the fluorescence intensity of pig liver-based CQDs is related to $\mathrm{pH}$. In the range of $\mathrm{pH} 2.0-7.0$, the fluorescence intensity increased gradually and the fluorescence quenching efficiency reached its maximum, but after $\mathrm{pH}$ 7.0, the fluorescence intensity decreased gradually. The results show that the fluorescence quenching response of the system is the largest at $\mathrm{pH} 7.0$, according to which $\mathrm{pH} 70$ is the optimum $\mathrm{pH}$ value of citric acid-disodium hydrogen phosphate buffer.

In order to study the stability of pig liver-based CQDs in detecting permanganate ions, the fluorescence intensity of pig liver-based CQDs in the presence of permanganate ions at the same concentration under different reaction time was studied. As shown in Figure 9B, the fluorescence intensity of pig liver based-CQDs decreased sharply within 0-1 min and gradually within 1-10 min, then tended to be stable, and reached the lowest fluorescence intensity in $2 \mathrm{~min}$. Therefore, the reaction time of $2 \mathrm{~min}$ was chosen as the optimal fluorescence quenching response time. 

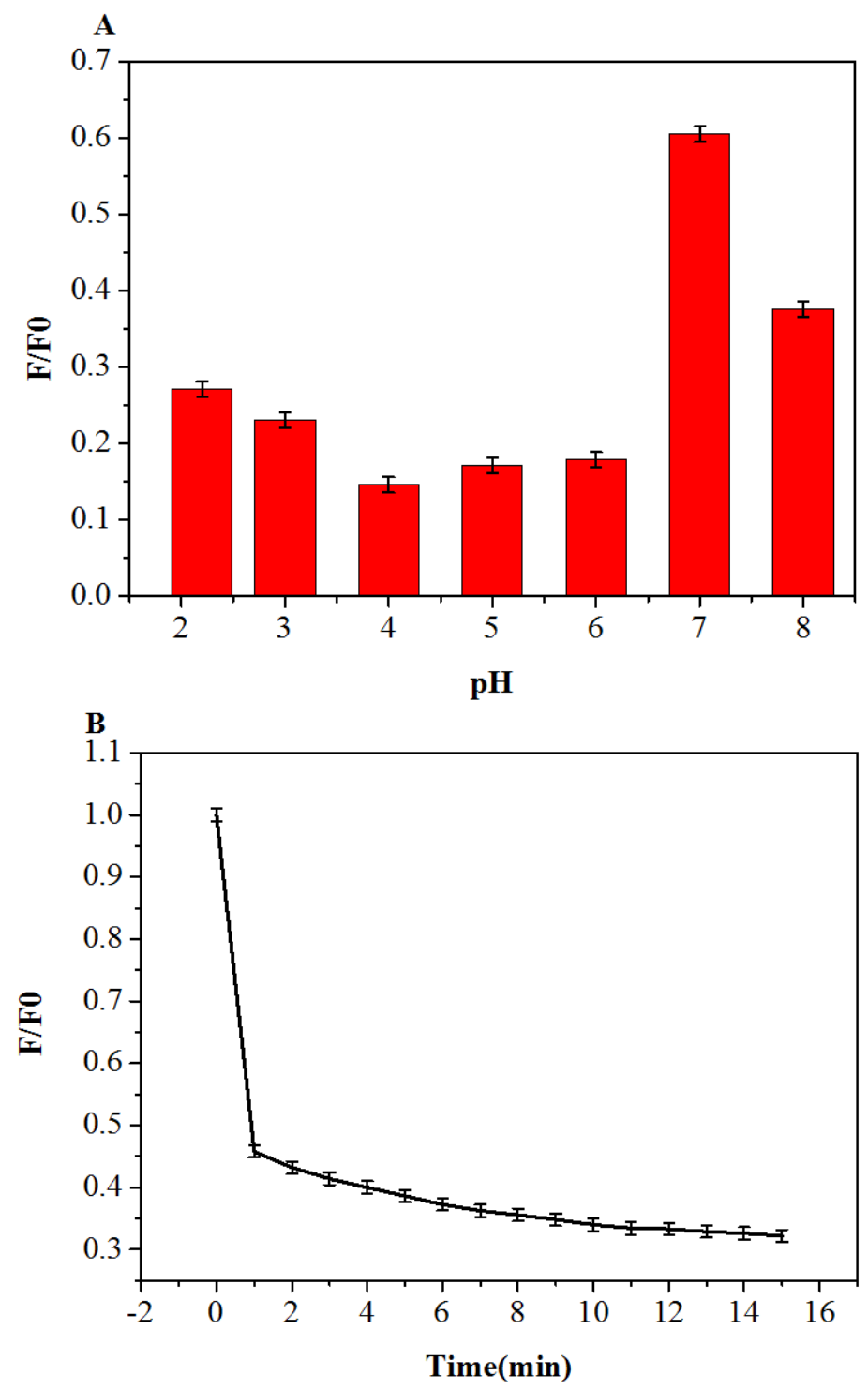

Figure 9. Effect of $p H(A)$ and $(B)$ reaction time on the fluorescence intensity of the pig liverbased CQDs

\section{Method performance study}

Under the optimal experimental conditions, the relationship between the fluorescence quenching intensity of pig liver-based CQDs and the concentration of $\mathrm{MnO}_{4}{ }^{-}$was studied, and the quantitative analysis method for fluorescence quenching of $\mathrm{MnO}_{4}{ }^{-}$was established. As shown in Figure 10, the fluorescence intensity of pig liver-based CQDs decreases gradually with the addition of $\mathrm{MnO}_{4}{ }^{-}$of different concentrations, and shows regular changes. As shown in Figure 11, the ratio of fluorescence intensity of the system was linear with the concentration of $\mathrm{MnO}_{4}{ }^{-}$in the range of $0-50 \mu \mathrm{M}$ both before and after addition of pig liver-based CQDs. The regression equation is $\mathrm{F} / \mathrm{F} 0=0.0151 \mathrm{C}$ $-0.0112\left(R^{2}=0.9967\right)$, where $F$ and $F 0$ are the fluorescence intensity of pig liver-based CQDs in the presence or absence of $\mathrm{MnO}_{4}{ }^{-}$respectively and $\mathrm{C}$ is the concentration of $\mathrm{MnO}_{4}^{-}$. The detection limit of $\mathrm{MnO}_{4}^{-}$was $0.06 \mu \mathrm{M}$ and the signal-noise ratio was $3(\mathrm{~S} / \mathrm{N})$. 


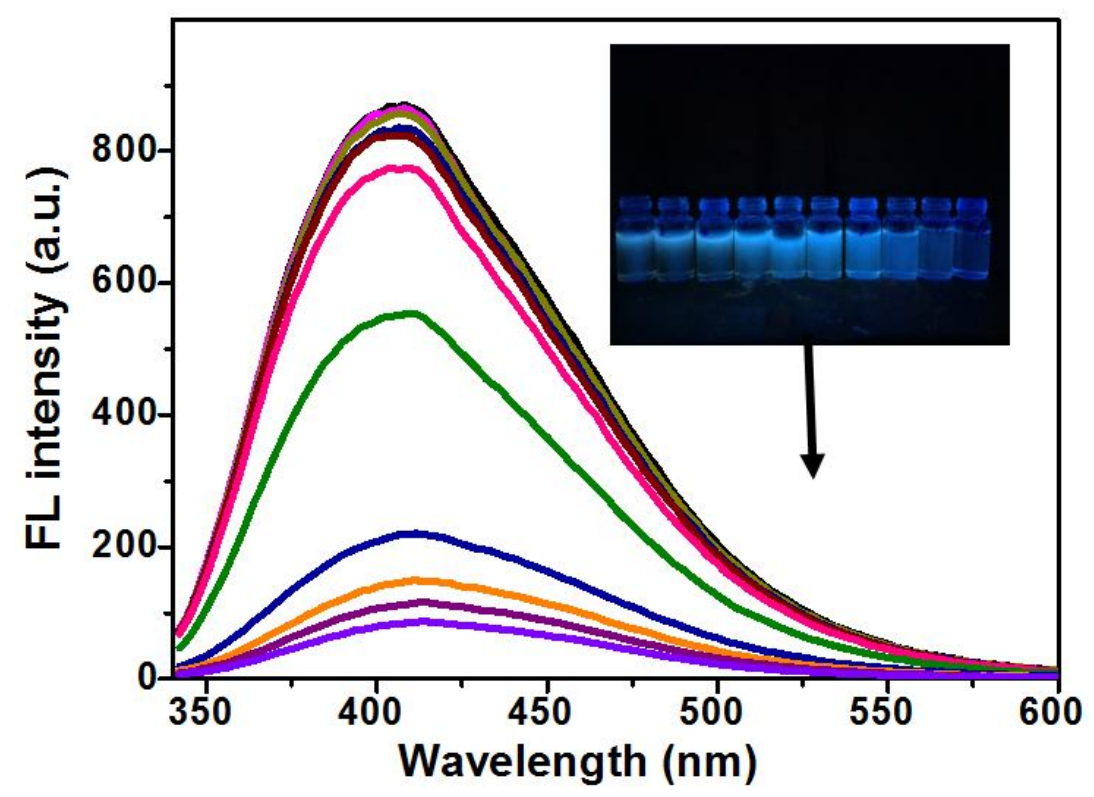

Figure 10. Fluorescence response of pig liver-based CQDs upon addition of different concentrations of $\mathrm{MnO}_{4}^{-}$(concentration gradient of pig liver based-CQDs under ultraviolet light)

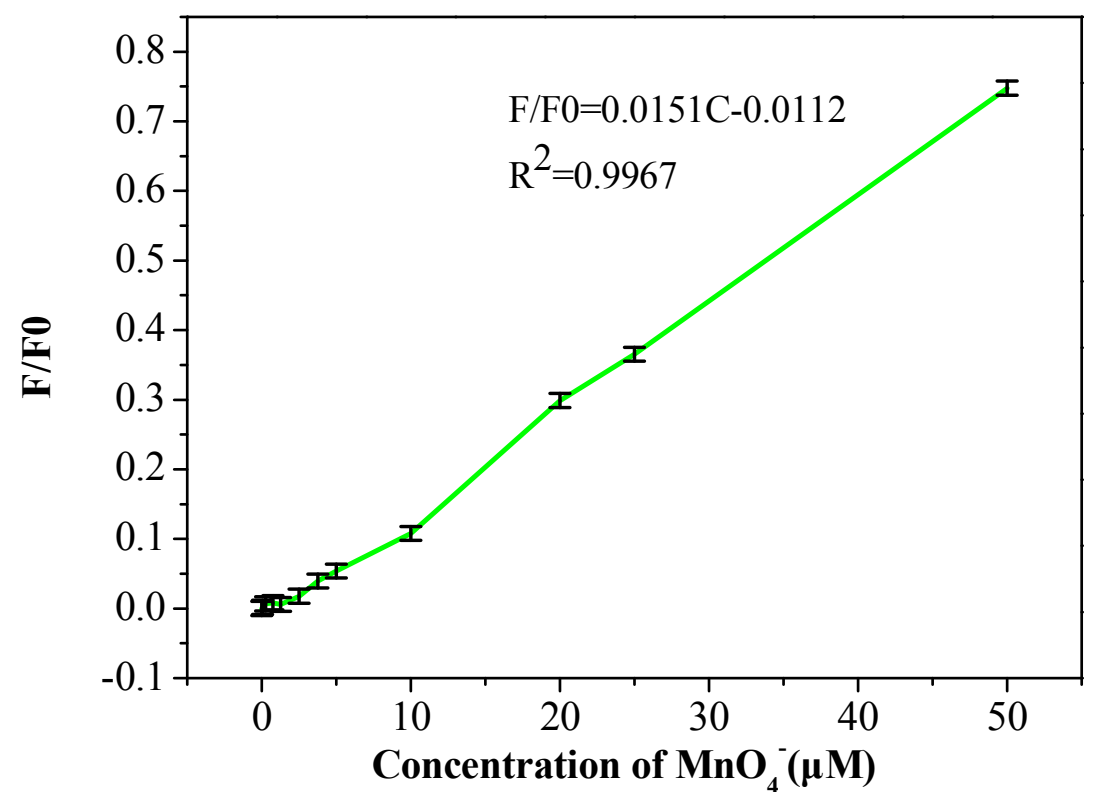

Figure 11. Linear correlation of $\mathrm{FO} / \mathrm{F}$ values versus the concentration of $\mathrm{MnO}_{4}^{-}$over the range from 0.1 to $50 \mu M$

\section{Possible fluorescence quenching mechanism}

In recent years, many sensors have been established based on the fluorescence variation of carbon quantum dots. These sensors were primarily analytes that quenched or increased the fluorescence of the carbon dots (Mariappan et al., 2017). Theoretically, this fluorescence phenomenon is related to different mechanisms. For fluorescence 
quenching, the mechanism is usually divided into dynamic quenching and static quenching, caused by the collision between fluorescent materials and quencher and the formation of ground state complexes respectively (Kaviyarasu et al., 2017). In case of dynamic quenching, the Stern-Volmer equation can be used for analysis (Guo et al., 2015) with the following formula:

$$
\mathrm{F} 0 / \mathrm{F}=1+\mathrm{K}_{\mathrm{q} 00}[\mathrm{Q}]=1+\mathrm{K}_{\mathrm{Sv}}[\mathrm{Q}]
$$

where: F0 and $\mathrm{F}$ indicate the fluorescence intensity before and after the addition of $\mathrm{MnO}_{4}{ }^{-}$respectively, $\mathrm{K}_{\mathrm{q}}$ is the bimolecular quenching constant, $\tau 0$ is the average lifetime of CQDs, generally $10^{-8} \mathrm{~s}, \mathrm{KSV}$ is the Stern-Walmer quenching constant, and [Q] was the concentration of $\mathrm{MnO}_{4}^{-}$. Therefore, the fluorescence quenching process may be caused by static quenching, i.e. the formation of ground state complexes in the reaction system (Lakowicz, 2006; Sun et al., 2015; Formica et al., 2012). The absorption strength of CQDs increased and the peak shifted to blue after addition of $\mathrm{MnO}_{4}{ }^{-}$, indicating that the interaction between $\mathrm{MnO}_{4}{ }^{-}$and $\mathrm{CQDs}$ produced the ground state complex. According to almost constant fluorescence lifetime, the fluorescence quenching caused by $\mathrm{MnO}_{4}{ }^{-}$may be static quenching due to the formation of stable nonfluorescent complexes (Zuo et al., 2016; Sun and Lei, 2017; Wu et al., 2011; Liu et al., 2017, 2014). FT-IR and XPS confirmed the presence of -COOH, -OH, etc. on the surface of CQDs, indicating good water solubility and good application prospect of the synthesized CQDs.

\section{Analysis of $\mathrm{MnO}_{4}^{-}$in real samples}

The above mentioned environmental water and soil samples were pretreated, and then $\mathrm{MnO}_{4}{ }^{-}$in environmental water and soil samples was detected by prepared pig liverbased CQDs. The environmental water samples and soil samples were spiked and recovered using different concentrations of $\mathrm{MnO}_{4}{ }^{-}$and pretreated according to the same procedure. As shown in Table 1, the recovery of $\mathrm{MnO}_{4}^{-}$in water samples is 94.2 102.5\% and the relative standard deviations (RSD) of all samples are less than $5.8 \%$. As shown in Table 2, the recovery of $\mathrm{MnO}_{4}{ }^{-}$in soil samples is 90.5 to $99.5 \%$ and the relative standard deviations (RSD) of all samples are below 4.7\%. Therefore, this method has the potential for detecting $\mathrm{MnO}_{4}{ }^{-}$in environmental water samples and soil samples.

Table 1. Analytical results of $\mathrm{MnO}_{4}^{-}$in the water samples $(n=3)$

\begin{tabular}{c|c|c|c|c}
\hline Samples & $\begin{array}{c}\text { Spiked } \\
(\boldsymbol{\mu g} / \mathbf{m L})\end{array}$ & $\begin{array}{c}\text { Found } \\
(\boldsymbol{\mu g} / \mathbf{m L})\end{array}$ & $\begin{array}{c}\text { Recovery } \\
(\mathbf{\%})\end{array}$ & $\begin{array}{c}\text { RSD } \\
(\mathbf{\%}, \mathbf{n}=\mathbf{3})\end{array}$ \\
\hline \multirow{3}{*}{ Tap water } & 0 & $\mathrm{ND}$ & - & - \\
& 5 & 4.71 & 94.2 & 4.3 \\
& 20 & 19.3 & 96.5 & 3.6 \\
\hline \multirow{3}{*}{ Lake water } & 0 & $\mathrm{ND}$ & - & - \\
& 5 & 4.92 & 98.4 & 5.8 \\
& 20 & 20.5 & 102.5 & 2.5 \\
\hline \multirow{2}{*}{ Waste water } & 0 & $\mathrm{ND}$ & - & - \\
& 5 & 5.10 & 102.0 & 5.3 \\
\hline
\end{tabular}

$\mathrm{ND}=$ not detected 
Table 2. Analytical results of $\mathrm{MnO}_{4}^{-}$in the soil samples $(n=3)$

\begin{tabular}{c|c|c|c|c}
\hline Samples & $\begin{array}{c}\text { Spiked } \\
(\boldsymbol{\mu g} / \mathbf{m L})\end{array}$ & $\begin{array}{c}\text { Found } \\
(\boldsymbol{\mu g} / \mathbf{m L})\end{array}$ & $\begin{array}{c}\text { Recovery } \\
(\mathbf{\%})\end{array}$ & $\begin{array}{c}\text { RSD } \\
(\mathbf{\%}, \mathbf{n}=\mathbf{3})\end{array}$ \\
\hline \multirow{3}{*}{ Sample 1 } & 0 & ND & - & - \\
& 5 & 4.97 & 99.5 & 4.5 \\
& 20 & 18.1 & 90.5 & 2.8 \\
\hline \multirow{3}{*}{ Sample 2 } & 0 & $\mathrm{ND}$ & - & - \\
& 10 & 9.58 & 95.8 & 3.5 \\
& 20 & 18.34 & 91.7 & 3.1 \\
\hline
\end{tabular}

$\mathrm{ND}=$ not detected

\section{Conclusion}

In summary, novel fluorescent CQDs were prepared from pig liver by hydrothermal synthesis method and the fluorescence intensity of CQDs could be quenched by $\mathrm{MnO}_{4}{ }^{-}$, so the CQDs were used as nanosensors for the sensitive and selective detection of $\mathrm{MnO}_{4}^{-}$in environmental samples. For instance, morphology, particle size, crystal form, chemical element composition and optical properties of the prepared pig liver-based CQDs were characterized by TEM, FT-IR, XPS, UV-Vis and FS. The results showed that it had good stability and fluorescence characteristics. In addition, a good linear relationship was achieved between the fluorescence response of CQDs and the concentration of $\mathrm{MnO}_{4}{ }^{-}$in the range from 0.1 to $50 \mu \mathrm{M}$. The detection limit was $0.06 \mu \mathrm{M}$ under optimized conditions. The developed method has been successfully applied to the determination of $\mathrm{MnO}_{4}^{-}$in water samples and soils of environmental samples. This method is green, environmental friendly, simple and easy to operate, and shows great potential for application.

\section{REFERENCES}

[1] Bhunia, S. K., Saha, A., Maity, A. R., Ray, S. C., Jana, N. R. (2013): Carbon nanoparticle-based fluorescent bioimaging probes. - Sci. Rep. 3: 1473.

[2] Clarkson, T. W., Magos, L. (2006): The toxicology of mercury and its chemical compounds. - Crit. Rev. Toxicol. 36(8): 609-662.

[3] da Silva, E. G. P., do N. Santos, A. C., Costa, A. C. S., da N. Fortunato, D. M., José, N. M., Korn, M. G. A., dos Santos, W. N. L., Ferreira, S. L. C. (2006): Determination of manganese and zinc in powdered chocolate samples by slurry sampling using sequential multi-element flame atomic absorption spectrometry. - Microchemical Journal 82(2): 159-162.

[4] Deng, X. Y., Li, J. Y., Tan, K. J. (2014): Rapid screening and confirmation of pesticide residues in potato by high-resolution benchtop Q exactive LC-MS. - Chinese J. Anal. Chem. 42(4): 579-584.

[5] Doroschuk, V. O., Lelyushok, S. O., Ishchenko, V. B., Kulichenko, S. A. (2004): Flame atomic absorption determination of manganese (II) in natural water after cloud point extraction. - Talanta 64(4): 853-856.

[6] Formica, M., Fusi, V., Giorgi, L., Micheloni, M. (2012): New fluorescent chemosensors for metal ions in solution. - Coord. Chem. Rev. 256 170-192.

[7] Gunter, T. E., Miller, L. M., Gavin, C. E., Eliseev, R., Salter, J., Buntinas, L., Alexandrov, A., Hammond, S., Gunter, K. K. (2010): Determination of the oxidation states of manganese in brain, liver, and heart mitochondria. - Journal of Neurochemistry 88(2): 266-280. 
[8] Guo, Y., Zhang, L., Zhang, S., Yang, Y., Chen, X., Zhang, M. (2015): Fluorescent carbon nanoparticles for the fluorescent detection of metal ions. - Biosens. Bioelectron. 63 6171.

[9] Hua, X.-W., Bao, Y.-W., Wang, H.-Y., Chen, Z., Wu, F.-G. (2017): Bacteria-derived fluorescent carbon dots for microbial live/dead differentiation. - Nanoscale 9: 2150-2161.

[10] Jones, K. H., Senft, J. A. (1985): An improved method to determine cell viability by simultaneous staining with fluorescein diacetate-propidium iodide. - J. Histochem. Cytochem. 33: 77-79.

[11] Kaviyarasu, K., Kanimozhi, K., Matinise, N., Maria, C. Magdalane, Mola, G. T., Kennedy, J., Maaza, M. (2017): Antiproliferative effects on human lung cell lines A549 activity of cadmium selenide nanoparticles extracted from cytotoxic effects: Investigation of bio-electronic application. - Mater. Sci. Eng. C. 76 1012-1025.

[12] Lakowicz, J. R. (2206): Principles of Fluorescence Spectroscopy. 3rd. Ed. - Springer, Singapore.

[13] Li, H., Kong, W. Q., Liu, J., Liu, N. Y., Huang, H., Liu, Y., Kang, Z. H. (2015): Fluorescent N-doped carbon dots for both cellular imaging and highly-sensitive catechol detection. - Carbon 91: 66-75.

[14] Li, H. T., Kang, Z. H, Liu, Y. et al. (2012): Carbon nanodots: synthesis, properties and applications. - J. Mater. Chem. 22(46): 24230-24253.

[15] Li, J. Z., Wang, N. Y., Tran, T. T. et al. (2013): Electrogenerated chemiluminescence detection of trace level pentachlorophenol using carbon quantum dots. - Analyst 138(7): 2038-2043.

[16] Li, Z., Yu, H. J., Bian, T., Zhao, Y. F., Zhou, C., Shang, L., Liu, Y. H., Wu, L. Z., Tung, C. H., Zhang, T. R. . (2015): Highly luminescent nitrogen-doped carbon quantum dots as effective fluorescent probes for mercuric and iodide ions. - Mater. Chem. C 3: 19221928.

[17] Liang, P., Sang, H., Sun, Z. (2006): Cloud point extraction and graphite furnace atomic absorption spectrometry determination of manganese (II) and iron(III) in water samples. Journal of Colloid \& Interface Science 304(2): 486-490.

[18] Lim, S. Y., Shen, W., Gao, Z. Q. (2015): - Chem. Soc. Rev. 44: 362.

[19] Liu, Y., Zhao, Y., Zhang, Y. (2014): One-step green synthesized fluorescent carbon nanodots from bamboo leaves for copper(II) ion detection. - Sens. Actuators B 196: 647652.

[20] Liu, Y., Duan, W., Song, W., Liu, J., Ren, C., Wu, J., Liu, D., Chen, H. (2017): Red emission $\mathrm{B}, \mathrm{N}, \mathrm{S}$-co-doped carbon dots for colorimetric and fluorescent dual mode detection of $\mathrm{Fe} 3+$ ions in complex biological fluids and living cells. - ACS Appl. Mat. Interfaces 9(14): 12663-12672.

[21] Lu, W., Qin, X., Liu, S. et al. (2012): Economical, green synthesis of fluorescent carbon nanoparticles and their use as probes for sensitive and selective detection of mercury(II) ions. - Analytical Chemistry 84(12): 5351-5357.

[22] Mariappan, A., Kaviyarasu, K., Neyvasagam, K., Ayeshamariam, A., Pandi, P., Palanichamy, R. R., Gopinathan, C., Mola, G. T., Maaza, M. (2017): - Surf. Interface. 6 247-255.

[23] Ming, H., Ma, Z., Liu, Y., Pan, K. M., Yu, H., Wang, F., Kang, Z. H. (2012): Large scale electrochemical synthesis of high quality carbon nanodots and their photocatalytic property. - Dalton Trans. 41: 9526-9531.

[24] Nocker, A., Cheung, C. Y., Camper, A. K. (2006): Comparison of propidium monoazide with ethidium monoazide for differentiation of live vs. dead bacteria by selective removal of DNA from dead cells. - J. Microbiol. Methods 67(2): 310-320.

[25] Rask, J. H., Miner, B. A. Buseck, P. R. (2015): Determination of manganese oxidation states in solids by electron energy-loss spectroscopy. - Ultramicroscopy 21(4): 321-326.

[26] Sun, J., Yang, S. W., Wang, Z. Y., Shen, H., Xu, T., Sun, L. T., Li, H., Chen, W. W., Jiang, X. Y., Ding, G. Q., Kang, Z. H., Xie, X. M., Jiang, M. H. (2015): Ultra-high 
quantum yield of graphene quantum dots: aromatic-nitrogen doping and photoluminescence mechanism. - Part. Part. Syst. Charact. 32: 434-440.

[27] Sun, X., Lei, Y. (2017): Fluorescent carbon dots and their sensing applications. - Trends in Analytical Chemistry 89 163-180.

[28] Sun, X., Wang, Y., Lei, Y. (2015): Fluorescence based explosive detection: from mechanisms to sensory materials. - Chem. Soc. Rev. 44 8019-8061.

[29] Teo, K. C., Chen, J. (2001): Determination of manganese in water samples by flame atomic absorption spectrometry after cloud point extraction. - Analyst 126(4): 534.

[30] Wang, Y. F., Hu, A. G. (2014): Carbon quantum dots: synthesis, properties and applications. - J. Mater. Chem. C 2(34): 6921-6939.

[31] Wu, J., Liu, W., Ge, J., Zhang, H., Wang, P. (2011): New sensing mechanisms for design of fluorescent chemosensors emerging in recent years. - Chem. Soc. Rev. 40: 3483-3495.

[32] Xu, X. Y., Robert, R., Gu, Y. L. et al. (2004): Electrophoretic analysis and purification of fluorescent single-walled carbon nanotube fragments. - J. am. Chem. Soc. 126(40): 12736-12737.

[33] Yu, H. J., Shi, R., Zhao, Y. F., Waterhouse, G. I. N., Wu, L. Z., Tung, C. H., Zhang, T. R. (2016): Nitrogen-doped porous carbon nanosheets templated from g-C3 N4 as metal-free electrocatalysts for efficient oxygen reduction reaction. - Adv. Mater. 28(25): 9454-9477.

[34] Zeng, C., Qin, P., Lan, L., Wei, H., Wu, W. (2017): Chemical vapor generation coupled with atomic fluorescence spectrometry for the determination of manganese in food samples. - Microchemical Journal 131: 31-35.

[35] Zhang, R., Chen, W. (2014): Nitrogen-doped carbon quantum dots: facile synthesis and application as a "turn-off" fluorescent probe for detection of $\mathrm{Hg}^{2+}$ ions. - Biosensors and Bioelectronics 55: 83-90.

[36] Zhao, H. X., Liu, L. Q., Liu, Z. D. et al. (2011): Highly selective detection of phosphate in very complicated matrixes with an off-on fluorescent probe of europium-adjusted carbon dots. - Chem. Commun. 47(9): 2604-2606.

[37] Zuo, P., Lu, X., Sun, Z., Guo, Y., He, H. (2016): A review on syntheses, properties, characterization and bioanalytical applications of fluorescent carbon dots. - Microchim. Acta 183 519-542. 